\title{
A new approach for the design of plates with trapezoidal longitudinal stiffeners
}

\author{
M.Z. Haffar \& S. Ádány \\ Department of Structural Mechanics, Budapest University of Technology, Budapest, Hungary \\ B. Kövesdi \\ Department of Structural Engineering, Budapest University of Technology, Budapest, Hungary
}

\begin{abstract}
The design approach for longitudinally stiffened plates in the relevant Eurocode interprets the stiffener buckling as a combination of so-called plate-like behavior and column-like behavior. In this paper an alternative design approach is presented for the problem of compressed plates with longitudinal trapezoidal stiffeners. One single Winter-type buckling curve is proposed for the calculation of the reduction factor for stiffener buckling. The proposed buckling curve is described by one single formula, but with some geometrydependent parameters. The parameters are determined and the results are evaluated by large number of advanced finite element analyses.
\end{abstract}

\section{INTRODUCTION}

The current European design approach for the resistance calculation of longitudinally stiffened plates can be found in CEN (2006). It assumes that, depending on the geometry, the behavior can be column-like, plate-like, or in between the plate-like and column-like behavior modes, i.e. interactive. To calculate the resistance, a reduction factor is to be determined, separately for the column-like and plate-like behaviors. For the reduction factor for the column-like behavior an Ayrton-Perry formula is used, as usual in the European practice for column design. For the reduction factor for the plate-like behavior a Winter formula is used, as usual in the European practice for plate design. In a general case the final reduction factor is interpolated from the two separate reduction factors. Once the final $\rho_{c}$ reduction factor is known, the compression resistance of the stiffened plate is calculated by Equation (1).

$$
N_{R d}=\frac{f_{y}\left(\rho_{c} \cdot A_{c, e f f, l o c}+A_{e d g e, e f f}\right)}{\gamma_{M 0}}
$$

where $A_{c, \text { eff, loc }}$ is the effective area of the entire internal part of the stiffened plate, $A_{\text {edge,eff }}$ is the total effective area of the edge plate elements, see Figure 1, while $\gamma_{M 0}$ is a partial factor and $f_{y}$ is the yield strength.

The precision of the above design approach has recently been questioned, and proposals for various improvements in the design process have also been published, see e.g. Sinur (2011), Martin et al. (2017), or Kövesdi (2019). In this paper we propose a new approach, which is based on slightly different principles compared to the earlier proposals. 


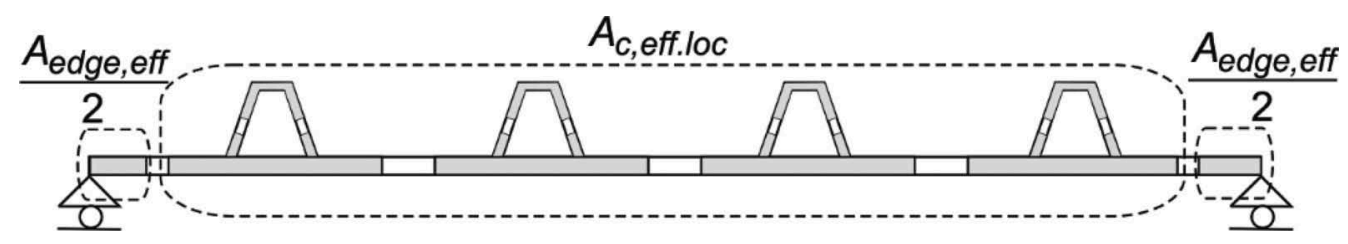

Figure 1. Separation of the compressed plate into middle and edge parts.

\section{PROPOSED NEW DESIGN METHOD}

The new design approach is originated from the observation that in cold-formed steel structural members the buckling of longitudinal stiffeners is usually described as distortional buckling, and in the various relevant cold-formed steel design codes the distortional buckling is covered by one single reduction factor, calculated by one single formula. The goal here, therefore, is to find a single formula for the reduction factor (instead of calculating it by interpolating from the reduction factors to plate-like and column-like behavior).

The proposed new design approach adopts Equation (1), as well as utilizes some formulae from the current design process. The main novelty is in the calculation of the reduction factor. The proposed design process can be completed through the following steps:

1. $\sigma_{c r, p}$ is the critical buckling stress must be calculated; it is recommended to perform shell finite element calculation (linear buckling analysis).

2. The non-dimensional slenderness $\bar{\lambda}_{p}$ is given by:

$$
\bar{\lambda}_{p}=\sqrt{\frac{f_{y}}{\sigma_{c r, p}}}
$$

3. The $\sigma_{c r, c}$ critical stress for a single stiffener is calculated as follows:

$$
\sigma_{c r, c}=\frac{\pi^{2} E I_{s l}}{a^{2} A_{s l}}
$$

where $I_{s l}$ is the second moment of area of the gross cross section of the stiffener and the adjacent parts of the plate, relative to the out-of-plane bending of the plate; $A_{s l}$ is the gross cross-sectional area of the stiffener and the adjacent parts of the plate, and $a$ is the buckling length (i.e. distance between the cross girders).

4. The $\xi$ stress ratio parameter, similarly as in CEN (2006), is given by:

$$
\xi=\frac{\sigma_{c r, p}}{\sigma_{c r, c}}-1
$$

5. The $\rho_{c}$ reduction factor is calculated by a new formula, as presented in Section 4 . 6. The $N_{R d}$ compression resistance of the stiffened plate is calculated by Equation (1).

It is to observe that the above procedure requires the calculation of the critical stress to the buckling where the whole stiffened panel buckles. Though this critical stress could be estimated by some analytical formula, but the numerical experience is that the available analytical formulae are not precise enough, see e.g. Haffar et al. (2019). That is why we propose to use shell finite element linear buckling analysis. 


\section{ADVANCED NUMERICAL ANALYSIS}

In order to find the new formula for the reduction factor (as in Step \#4 above), we would need to know the real resistance of various stiffened plates. The real resistance could ideally be obtained from real experiments, however, can be predicted by carefully completed advanced numerical analysis with considering geometric and material nonlinearity and imperfections (i.e. GMNI analyses). This latter approach is followed here. For the GMNIA the ANSYS 17.2 general purpose finite element software has been used, which has been used to calculate both the buckling resistance and the critical stress of the investigated panels. The model is described in detail and the results are reported in Haffar et al. (2019). Here only a brief summary is given.

The numerical model is a full shell model using four node thin shell elements (Shell 181 in Ansys terminology). All the sub-plates are divided into 6-10 elements in the transverse direction; the longitudinal discretization is adjusted to the transverse one in order to have nearly square elements. The model includes three panels, see Figure 2. The ends are supported by applying rigid constraints (see the left part of the figure). The longitudinal edges and the lines of the cross-girders are simply supported (see the right part of the figure). This model is a realistic representation of a typical application of stiffened plates such as flange element of a box-girder bridge.

The considered material is steel. A multi-linear elastic-plastic material model is applied. Global and local equivalent geometric imperfections have been used in the GMNI analyses with an amplitudes of $\min (a / 400, b / 400)$ for the global and $b_{i} / 200$ for the local imperfections according to the recommendations of CEN (2006), where $a$ is the length, $b$ is the width of the stiffened plate, while $b_{i}$ is the width of the plate part between two adjacent stiffeners, see Figure 2.

The variable parameters are as follows: the length of the panel $(a)$, the number of longitudinal stiffeners $(n)$, the distance between the longitudinal stiffeners $(w)$, the width and thickness of the analysed plate $\left(b, t_{p}\right)$ and the cross-section of the longitudinal stiffeners $\left(h_{s l}, t_{s l}\right.$, $\left.b_{s l, 1}, b_{s l, 2}\right)$, as shown in Figure 3. Altogether 394 different configurations are considered. In each case the ultimate resistance was aimed to calculate. Due to the applied model, the failure always occurred in the middle panel.

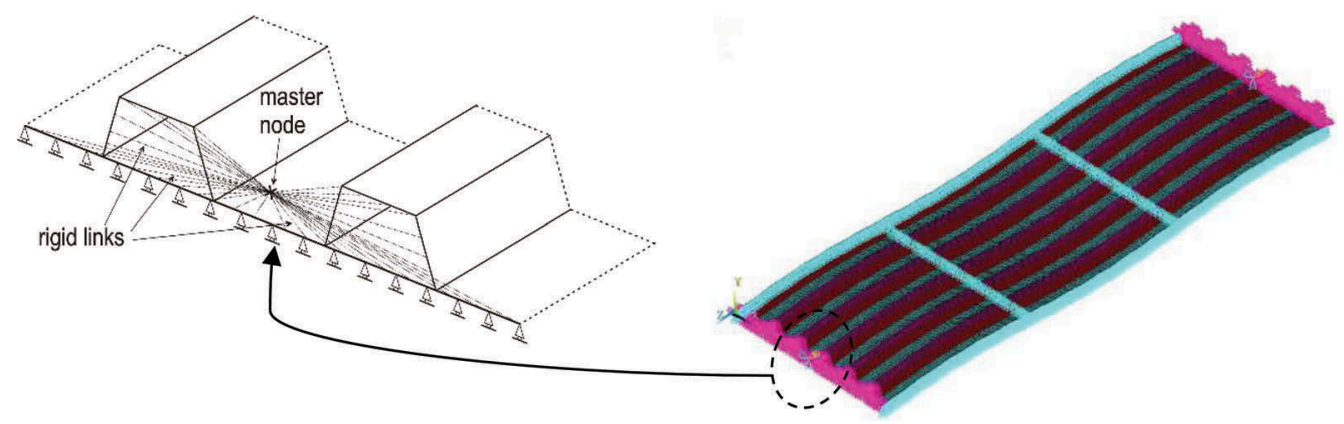

Figure 2. Applied shell model with the imperfections, and with the end supports highlighted.

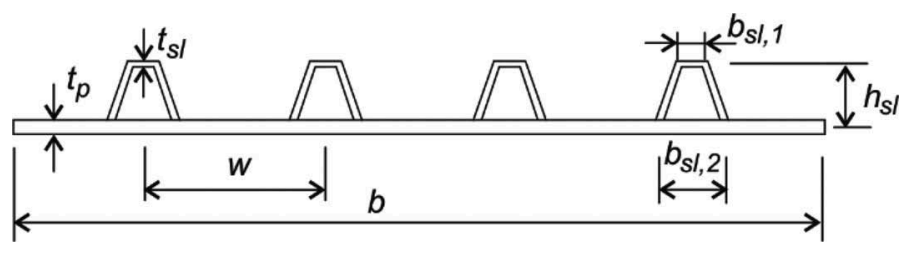

Figure 3. Geometric parameters in the parametric studies. 
Once the $N_{u l t}$ compression resistance of the stiffened plate is determined from the GMNIA, the reduction factor $\rho_{c, G M N I}$ can be back-calculated by rearranging Equation (1) to Equation (5).

$$
\rho_{c, G M N I}=\frac{N_{u l t} / f_{y}-A_{\text {edge }, e f f}}{A_{c, e f f, l o c}}
$$

It is to note that the geometric parameters were selected so that all the cross-sections would (at least approximately) be fully effective, thus, in the calculation of the relative slenderness parameter no adjustment for the effective cross-section is necessary in Step \#2 in Section 2. In case of not fully effective cross-sections Equation (2) could be modified.

\section{AUXILIARY MODIFIED WINTER CURVES}

The new design approach is based on the idea that the buckling of the stiffeners can be described as distortional buckling, and in the case of distortional buckling the reduction factor is calculated by Winter-type buckling curves in several design codes. Accordingly, some Winter-type buckling curves have been considered in our investigations, and finally a simple formula was found to be the most promising, as follows:

$$
\rho_{c}=\frac{\bar{\lambda}_{p}-\bar{\lambda}_{p 0}+1}{\left(\bar{\lambda}_{p}-\bar{\lambda}_{p 0}+1\right)^{e}} \text { if } \bar{\lambda}_{p}>\bar{\lambda}_{p 0}, \text { otherwise } \rho_{c}=1
$$

where $\bar{\lambda}_{p 0}$ and $e$ are two parameters that need to be determined. (It is to notice that $\bar{\lambda}_{p 0}$ has a physical meaning: it is the length of the plateau of the buckling curve.) The observation was that these two parameters are dependent on the $\xi$ stress ratio value. Therefore, the results from the 394 GMNI analyses were ordered into groups based on the stress ratio, and then the parameter values have been determined to each group. This was done by an optimization procedure, in which the difference between the $\rho_{\mathrm{c}, G M N I}$ reduction factor from GMNI analysis and the $\rho_{\mathrm{c}}$ reduction factor from Equation (6) was minimized. This minimization is completed range by range, leading to quasi-optimal $\bar{\lambda}_{p 0}$ and $e$ values for each $\xi$ range. The calculated parameters values are summarized in Table 1. In Figure 4 the found new curves are plotted for some selected ranges, together with the back-calculated reduction factors.

\section{THE FINAL REDUCTION FACTOR}

As it is clear from Table $1, \bar{\lambda}_{p 0}$ and $e$ are dependent on the $\xi$ value, which is visible also from Figure 5, where the quasi-optimal parameters are plotted in the function of the stress ratio. Figure 5 suggests that the parameters are continuous functions of the logarithm of is $\xi$. This means that instead of taking the $\bar{\lambda}_{p 0}$ and $e$ from Table 1 for the various ranges, it is simpler

Table 1. Parameters $\bar{\lambda}_{p 0}$ and $e$ for various $\xi$ ranges.

\begin{tabular}{lllllllll}
\hline$\xi$ from & $\xi$ to & $\bar{\lambda}_{p 0}$ & $e$ & & $\xi$ from & $\xi$ to & $\bar{\lambda}_{p 0}$ & $e$ \\
\hline 0 & 0.2 & 0.509 & 2.994 & & 1.6 & 2.0 & 0.597 & 2.138 \\
0.2 & 0.4 & 0.526 & 2.824 & & 2.0 & 2.5 & 0.550 & 2.099 \\
0.4 & 0.6 & 0.512 & 2.604 & & 2.5 & 3.0 & 0.562 & 2.052 \\
0.6 & 0.8 & 0.514 & 2.537 & & 3.0 & 4.0 & 0.591 & 2.116 \\
0.8 & 1.0 & 0.505 & 2.421 & & 4.0 & 5.0 & 0.667 & 2.229 \\
1.0 & 1.2 & 0.495 & 2.355 & & 5.0 & 10 & 0.728 & 2.385 \\
1.2 & 1.4 & 0.531 & 2.272 & & 10 & 25 & 0.937 & 2.664 \\
1.4 & 1.6 & 0.521 & 2.144 & & & & \\
\hline
\end{tabular}



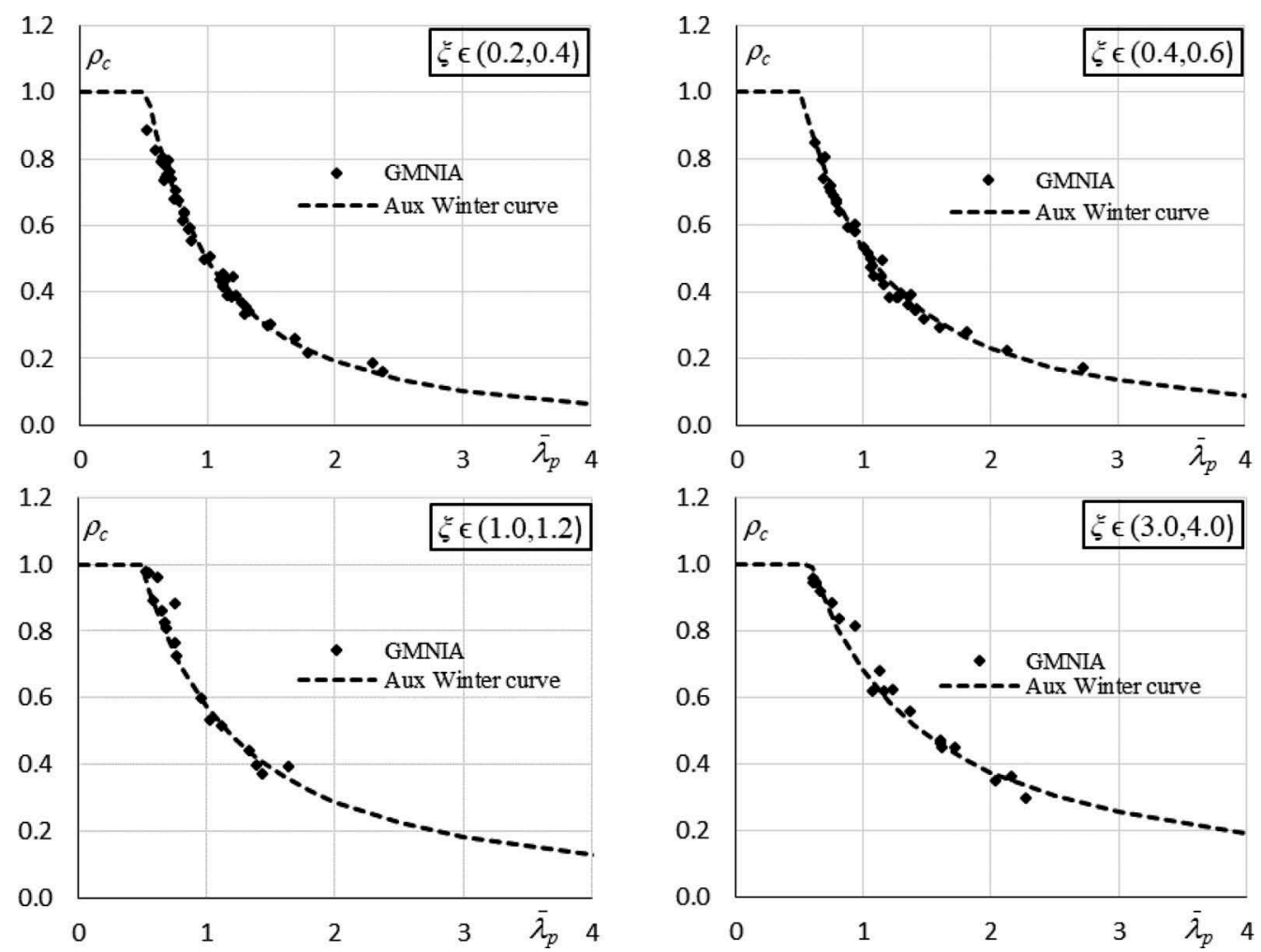

Figure 4. Auxiliary modified Winter curves and GMNIA reduction factors for 4 ranges of $\xi$.
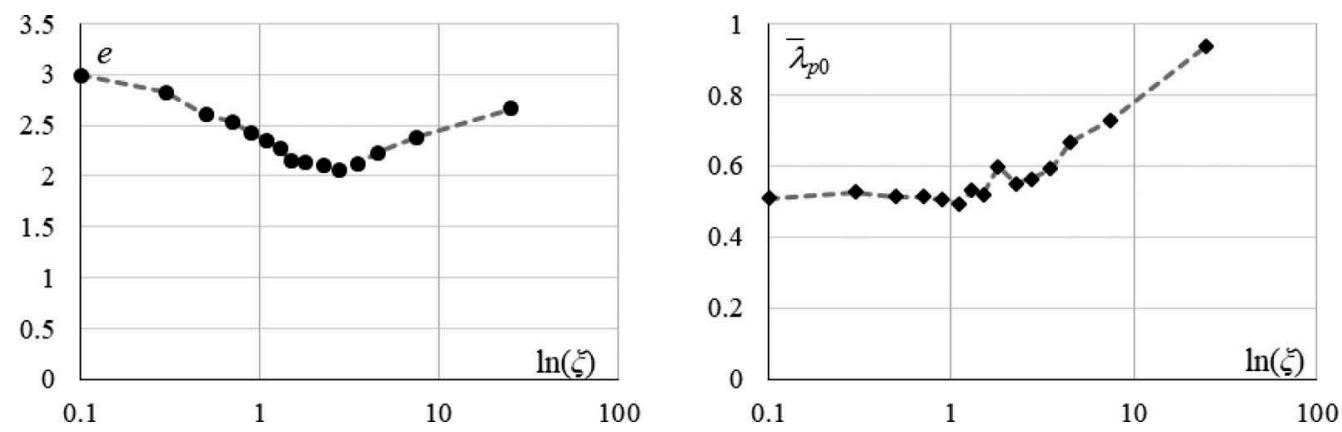

Figure 5. $\bar{\lambda}_{p 0}$ and $e$ values in the function of the $\xi$ stress ratio.

and potentially more precise to express them by analytical functions. Based on Figure 5, we assume here that both parameters can be approximated by two straight lines (in the semilogarithmic diagram), hence, we use the following formulae:

$$
\begin{gathered}
e=\max \left(A_{1} \ln (\xi)+B_{1} ; A_{2} \ln (\xi)+B_{2}\right), \\
\bar{\lambda}_{p 0}=\max \left(C_{1} \ln (\xi)+D_{1} ; D_{2}\right)
\end{gathered}
$$

The altogether 7 pieces of $A, B, C$ or $D$ constants have been determined by optimization in which the sum of the absolute differences between the $\rho_{c, G M N I}$ reduction factors from GMNI 


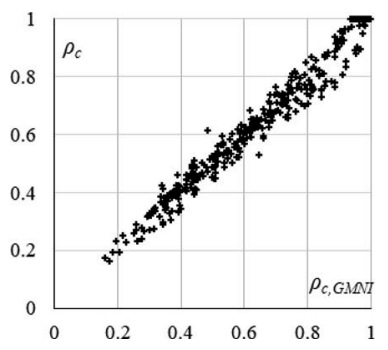

Figure 6. Comparison of the reduction factor values: GMNIA vs. Equations (6-8).

analysis and the $\rho_{c}$ reduction factors from Equations (6-8) is minimized. The final equations are as follows:

$$
\begin{aligned}
& e=\max \left\{\begin{array}{c}
-0.177 \ln (\xi)+2.411 \\
0.147 \ln (\xi)+1.862
\end{array}\right\}, \\
& \bar{\lambda}_{p 0}=\max \left\{\begin{array}{c}
0.488 \\
0.098 \ln (\xi)+0.526
\end{array}\right\} .
\end{aligned}
$$

\section{EVALUATION OF THE RESULTS}

All the cases (considered in the GMNI analyses) were re-calculated by using Equations (6-8), and the reduction factor values are compared to the GMNIA predictions. Figure 6 shows the comparison of the reduction factor values, while Figure 7 shows the $\left(\rho_{c, G M N I}-\rho_{c}\right) / \rho_{c, G M N I}$ relative differences in the function of the slenderness parameter. The most important statistical characteristics of the results are as follows:

- The average of the absolute values of the $\left(\rho_{c, G M N I}-\rho_{c}\right)$ differences is 0.030 .

- The mean value of the $\left(\rho_{c, G M N I}-\rho_{c}\right) / \rho_{c, G M N I}$ relative differences is $-0.2 \%$.

- The standard deviation of the $\left(\rho_{c, G M N I}-\rho_{c}\right) / \rho_{c, G M N I}$ relative differences is $6.8 \%$.

\section{CONCLUSION}

In this paper a new approach was proposed for the calculation of the resistance of longitudinally stiffened plates with trapezoidal stiffeners. The proposed approach adopts certain formulae from the current Eurocode procedure, still, it is theoretically different from the current

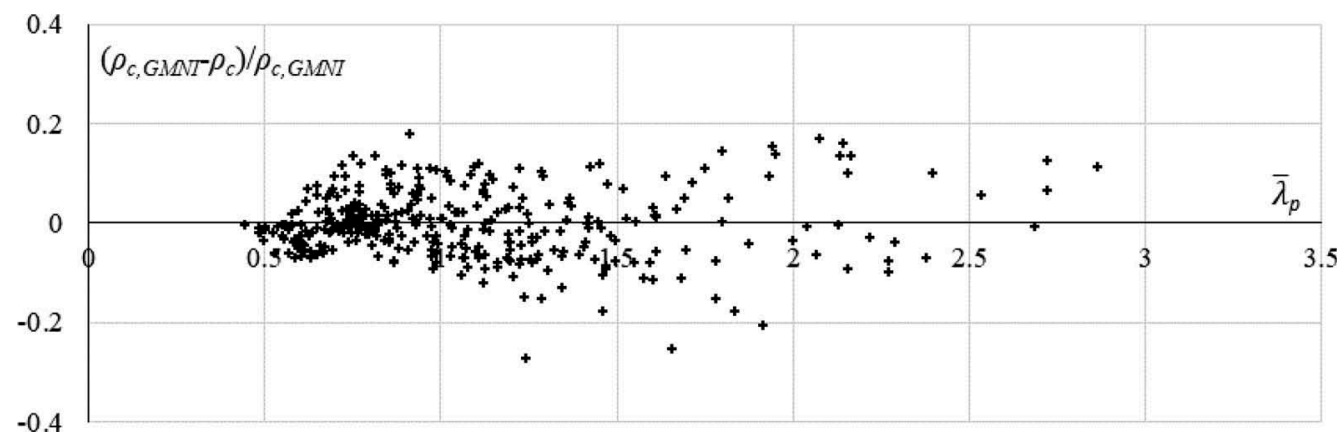

Figure 7. $\left(\rho_{c, G M N I}-\rho_{c}\right) / \rho_{c, G M N I}$ relative differences in the function of the slenderness parameter. 
Eurocode approach, since this latter one interprets the stiffener buckling as a combination of plate-like and column-like behavior, while in the new proposal no such interpretation is included. Accordingly, in the new design approach one single formula is applied to calculate one single reduction factor (which should be applied to reduce the cross-section resistance). The proposed formula is a modified Winter-formula.

The parameters of the proposed new formulae were determined by using the results of large number of resistance predictions by advanced finite element analyses. The results of the newly proposed design process were compared to those from the advanced numerical analyses, and they were evaluated statistically. Based on the statistical evaluation the new proposal was found to be reasonably precise.

It is to note that the reported research is still in progress. Other possible curves (including Ayrton-Perry type curves) are under consideration. Moreover, further geometries (including different types of longitudinal stiffeners) are intended to study in order to further enlarge the resistance database. The further results of the research are expected to be reported in the near future.

\section{ACKNOWLEDGEMENTS}

The presented research program was supported by the $\exists$ UnNP-18-4 New National Excellence Program of the Ministry of Human Capacities, by the János Bolyai Research Scholarship of the Hungarian Academy of Sciences and by the K119440 project of the Hungarian National Research, Development and Innovation Office. The financial supports are gratefully acknowledged.

\section{REFERENCES}

CEN 2006. EN 1993-1-5:2005, Eurocode 3: Design of steel structures, Part 1-5: Plated Structural elements.

Sinur F. 2011. Behavior of longitudinally stiffened girders under combination of high bending and shear loading, PhD thesis, University of Ljubljana, Faculty of Civil and Geodetical Engineering.

Martin P.O., Nguyen T.M., \& Davaine L. 2017. Effect the torsional stiffness of closed section stiffeners on plate buckling in Eurocode 3 Part 1-5, Research report, ECCS-TWG8.3, 2017.

Kövesdi B. 2019. Buckling resistance of orthotropic plates subjected by compression interpolation between plate and column-like behavior, Journal of Constructional Steel Research, Vol. 160, pp. 67-76.

Haffar M.Z., Kövesdi B. \& Ádány S. 2019. Buckling of longitudinally stiffened plates Part 2: Eurocode-based design for plate-like behavior of plates with closed-section stiffeners, Thin-Walled Structures, Vol. 145. 